\title{
An Evaluation of an Automated Detection Algorithm to Count Defects Present in X-Ray Topographical Images of SiC Wafers
}

Ian C. Brazil ${ }^{1}$, Patrick J. McNally ${ }^{1}$, Lisa O'Reilly ${ }^{1}$, Andreas Danilewsky ${ }^{2}$, Turkka O. Tuomi ${ }^{3}$, Aapo Lankinen ${ }^{3}$, Antti Säynätjoki ${ }^{3}$, Rolf Simon ${ }^{4}$, Stanislav Soloviev ${ }^{5}$, Larry B. Rowland ${ }^{5}$, and Peter M. Sandvik

${ }^{1}$ RINCE, Dublin City University, Dublin, 9, Ireland

${ }^{2}$ Kristallographisches Institute, Universität Freiburg, Freiburg, Germany

${ }^{3}$ Helsinki University of Technology, Helsinki, Finland

${ }^{4}$ Institut für Synchrotronstrahlung (ISS), Karlsruhe, Germany

${ }^{5}$ GE Global Research, Niskayuna, NY, 12309

\begin{abstract}
Full semiconductor wafer defect/dislocation characterization is difficult to implement manually. We present an analysis of an automated algorithm used to extract Threading Screw Dislocation defect data from Synchrotron White Beam X-Ray Topographical images of SiC wafers. This extraction involves a two-fold process; firstly the algorithm highlights the appropriate defect and secondly updates the counter to provide a final result of defect count. The result of the automated algorithm is compared to hand counts in all cases, thus allowing a critical analysis of the technique. Improvements to this algorithm have been made since last reported by the same authors [1], which are discussed. The analysis herein was also performed on a much larger sample of SiC wafer images than previously used by the same authors [1] allowing a better judgment of performance and critical evaluation. The algorithm is also compared with the original previous algorithm that was used [1]. The success of this methodology paves the way for a complete analysis of whole $\mathrm{SiC}$ wafers, which previously was extremely difficult due to image analysis inaccuracy or the bottleneck presented by manual counting.
\end{abstract}

\section{INTRODUCTION}

Silicon Carbide is a promising material for a variety of electronic applications. Properties $[3,8]$ include a large bandgap, high electrical breakdown, low chemical reactivity and a high operating temperature. Growth however is prone to defects, common defects include Micropipes, Basal Plane Dislocations (BPD), Stacking Faults (SF) and Threading Screw Dislocations (TSD) [2-4].

Synchrotron White Beam X-Ray Topography (SWXRT) can be used to analyze SiC wafers $[4,9,10]$ allowing a large image to be created of a wafer by joining smaller images. In SWXRT TSDs and BPDs [6] show up as dots and lines [5]. Being non-destructive its advantages over other techniques, e.g. $\mathrm{KOH}$ etching [7], are obvious. However quantitative analysis (e.g. TSD count) is extremely time consuming and tedious making SWXRT unsuited to examination of large quantities/areas of wafers. It is therefore a massive advantage if an automated method can be established to perform quantitative analysis of SWXRT images. An algorithm to classify Basal Plane Dislocations was discussed in a previous paper [1]. However the TSD algorithm developed in [1] was only semi-automatic. The complete automation of this algorithm and a demonstration of its usefulness is outlined in this paper. 


\section{EXPERIMENT}

The algorithm was trialed and evaluated empirically by using three methods defined as count measure, accuracy and difference of automated count to a manual hand count. The latter being a simple difference between a manual count and an automated count of TSDs, thus a positive number indicates an undercount, a negative number an overcount.

Accuracy and merit (good accuracy of determination) for the algorithm is defined by equation 1 below; this is the most valuable determinant as any algorithm must correctly identify a TSD.

$$
\text { Accuracy }=\frac{\text { Correctly Identified TSDs by algorithm }}{\text { Number of TSDs identified by algorithm }}
$$

Count measure is defined in equation 2 below although not as useful as accuracy because of the subjective nature of the manual count.

$$
\text { Count measure }=\frac{\text { Number of TSD s identified by Algorithm }}{\text { Number of TSD s identified by hand }}
$$

The count measure is defined as $100 \%$ if both manual and automated methods count zero TSDs and also if the algorithm determines a slightly higher number of TSDs compared to the manual count. Defined as $0 \%$ if the manual count identified zero TSDs and the algorithm determined greater than zero number of TSDs. V1.1 of the algorithm represents a modified (this being the addition of the high and low outputs) version of what was presented in [1]. V8 algorithm is the final version presented here for analysis. Both versions use similar methods to clean input threshold images e.g. typical TSD size. The main innovations in V8 are:

Scratch removal: Scratches resulted in large overcounts in V1.1. V8 removed these from the output by simple dilation of the clustered threshold output followed by subtraction of large objects from the image. This also helped remove micropipes, cracks and other artifacts.

Checking of Identified TSDs: For each dot the algorithm initially finds, it performs an automated check of the immediate region of this suspected TSD. If a suitable intensity gradient exists the probability of the dot found being a TSD increases. Thus the algorithm automatically rejects dots with low probability of being a TSD and accepts those with high probability. The gradient can be defined between 0 and 1 by the user.

Automated detection of ranges: V8 automatically determines the histogram profile of each image and chooses an appropriate point with which to perform initial thresholds.

Some 130 images from 8 different wafers are used to evaluate the algorithm's merits.

\section{DISCUSSION}

A 4H n-type Research Grade (RSH) $75 \mathrm{~mm}$ diameter $\mathrm{SiC}$ wafer is used to show the advantages of this new algorithm, figure 1 shows the difference in automated to manual count for both algorithms, V1.1 and V8. Version 8 represents a clear improvement. In total automatic mode Version 1.1 produces a massive overcount, it is not discussed further in this paper.

Figure 2 is a plot of the accuracy of the V8 algorithm as applied to 20 images from the wafer used in figure 1; the mean of the data is indicated by a dotted line i.e. $85 \%$. As can be seen the accuracy of the V8 algorithm for this wafer is extremely high. A high gradient of 0.95 was used. 


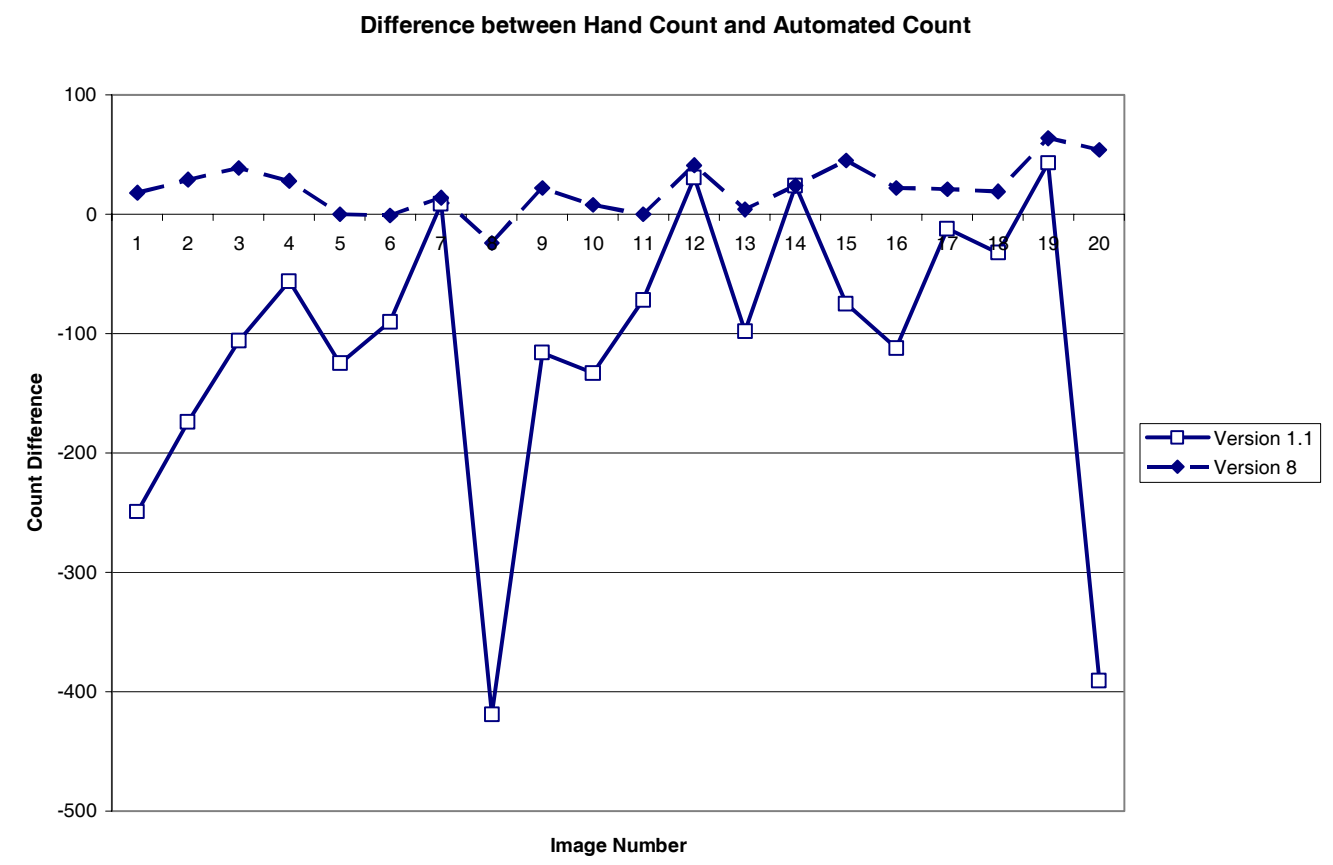

Figure 1. Difference between a Hand Count and Automated Count for old and improved algorithm

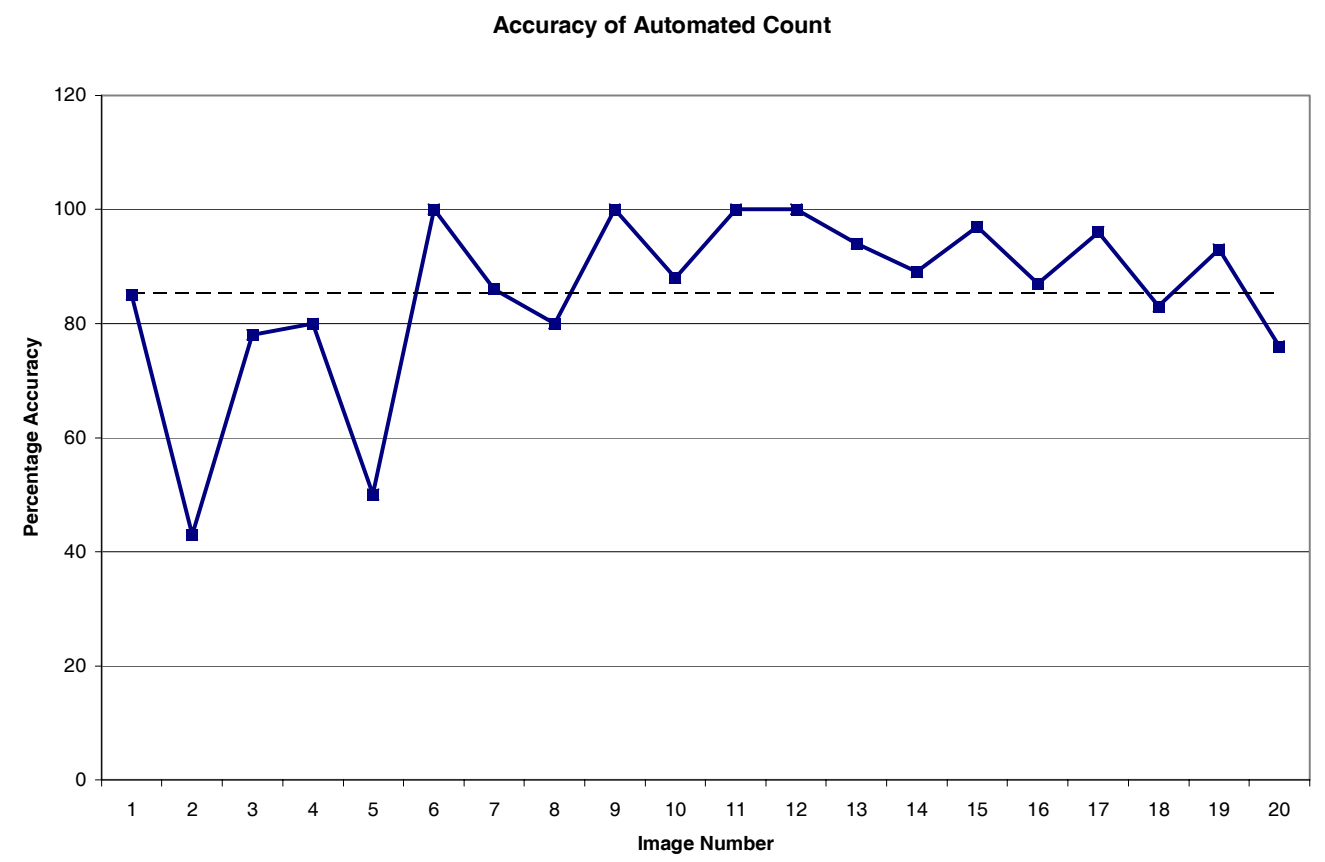

Figure 2. Percentage Accuracy of Automated Count for SiC 4H-n-RSH Grade Wafer

The average accuracy for approximately 130 images from 8 different wafers was determined and is plotted in Figure 3, the dotted line indicating the mean across all wafers. The accuracy remains extremely high. The exception is the $4 \mathrm{H}-\mathrm{p} \mathrm{SiC}$ wafer, wherein the relatively low accuracy is attributed to the lack of TSDs present on this wafer. If the algorithm detected 1 TSD where none existed in the image a value of $0 \%$ was awarded for accuracy. 
The average percentage of the count measure is shown in Figure 4 for the same images as used in Figure 3 with the dotted line indicating the mean across all 8 wafers. The count measure for the algorithm is relatively good, the exception again being the p-type $4 \mathrm{H} \mathrm{SiC}$ wafer the reason being the same for its relatively poor performance in accuracy.

Examples of the graphical output from the V8 algorithm are shown in Figure 5. TSDs are represented by the algorithm with a black circle surrounding them.

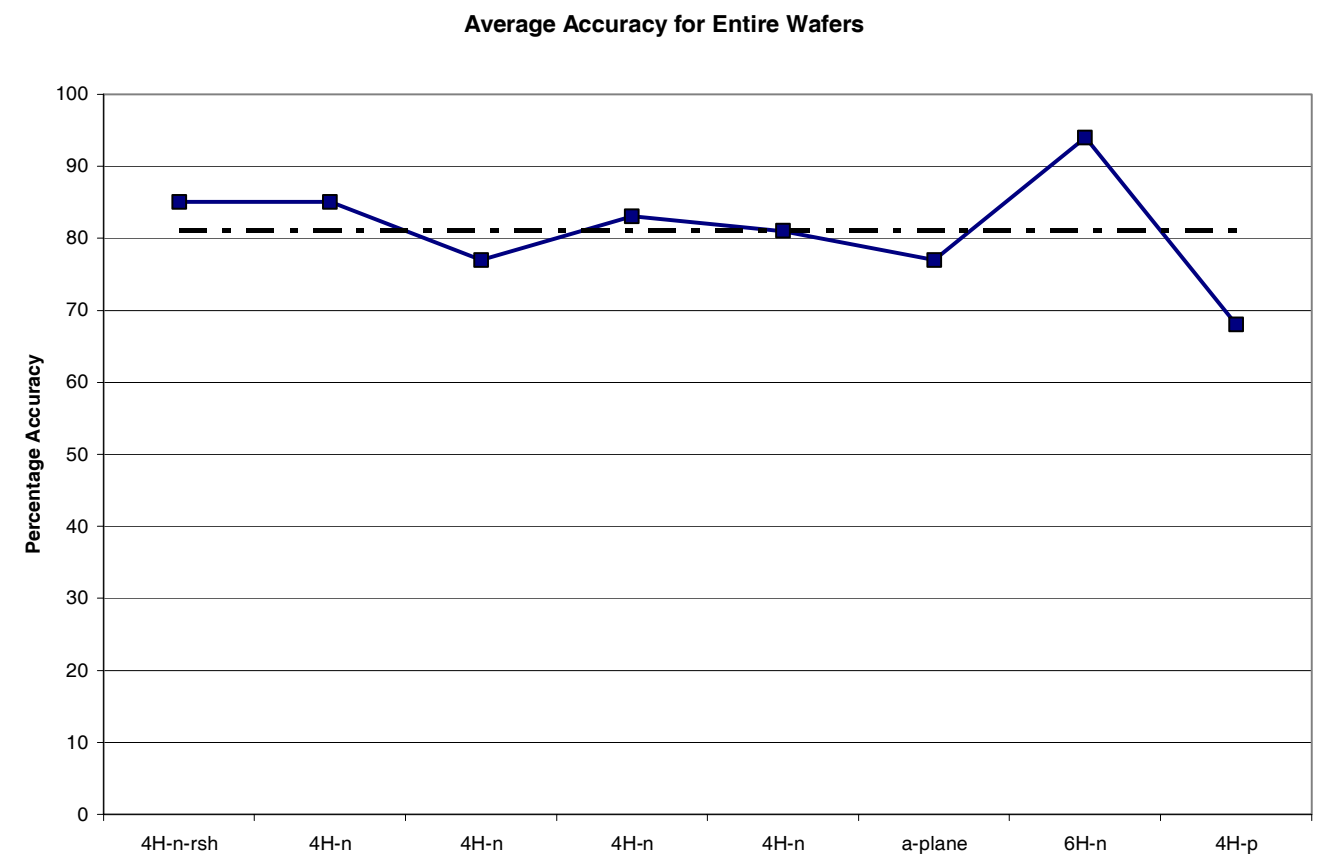

Figure 3. Percentage Average Accuracy across entire wafers (the different wafer types are indicated along the $\mathrm{x}$-axis) for which images are present

Count Measure

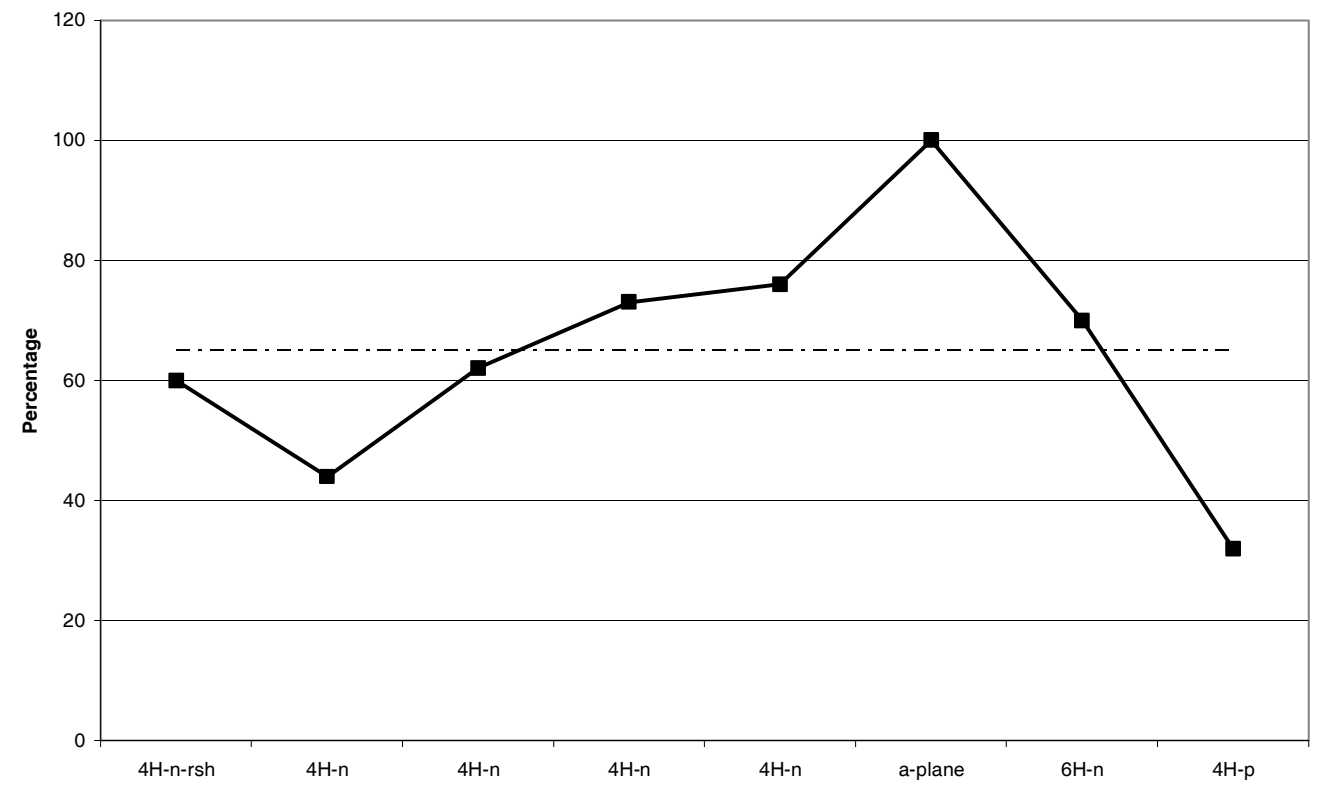

Figure 4. Count Measure Percentage 

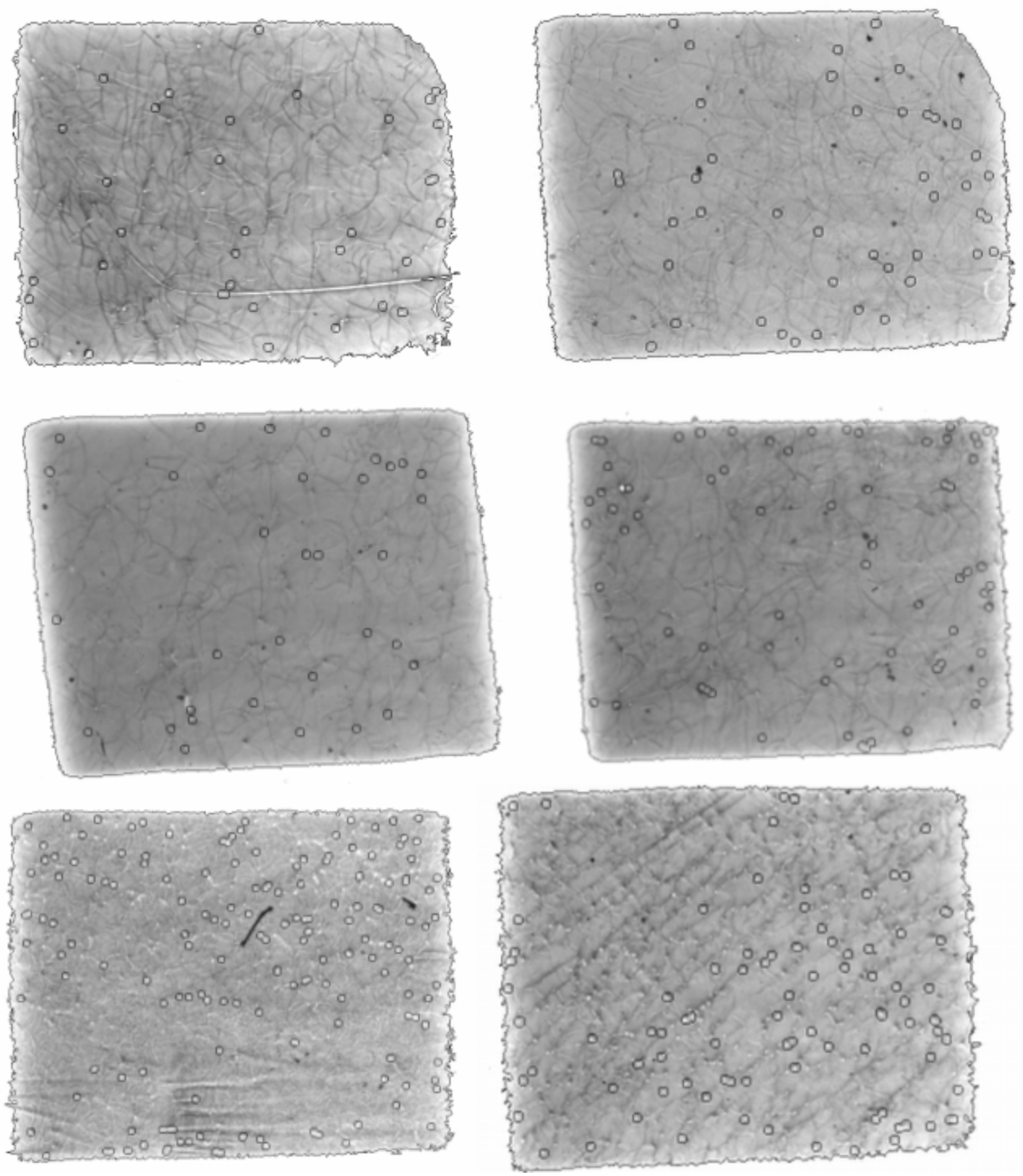

Figure 5. Examples of the graphical output of the algorithm.

Note the accurate identification of the TSDs, which appear as white dots in the x-ray. Basal plane dislocations (curved and tangled black lines), low angle grain boundaries (roughly parallel linear streaks) and the occasional scratch (e.g. long white line in the top LHS topograph) are not incorrectly marked as TSDs. Each topograph is approx $3 \mathrm{~mm} \times 2 \mathrm{~mm}$ in size. 


\section{CONCLUSIONS}

A full wafer defect or dislocation characterization using SWXRT cannot be implemented without an automated image processing and analysis of the captured topographic data. We have presented a novel image analysis procedure, which will allow the analysis of large numbers of semiconductor wafers for threading screw dislocations in $\mathrm{SiC}$, this accelerating $\mathrm{x}$-ray topographic characterization. The algorithm is highly accurate with an average count accuracy of $81 \%$ across a range of different types of $\mathrm{SiC}$ wafer.

There is also the possibility of improving count measure further. The finite threshold ranges that are used means it is not possible to capture every TSD. As a rough example, if threshold were to take place between 230-255 pixel intensities, any TSD outside this range would not be captured. This results in an ultimate upper limit on the detection algorithm as in some cases the TSD range is spread further than the chosen threshold ranges. One method not yet tested, but a potential solution to this problem, would be to extend the range limits so that more initial information is passed to the algorithm to define the TSDs. This should improve the count measure although the accuracy would be expected to remain roughly constant.

\section{REFERENCES}

1. I. Brazil , P.J. McNally, N. Ren, L. O’Reilly, A. Danilewsky, T.O. Tuomi, A. Lankinen, A. Säynätjaki, R. Simon, S. Soloviev, L. B. Rowland, P. M. Sandvik, Mater. Sci. Forum 556-557 (2007).

2. W. M. Vetter, M. Dudley, J. Appl. Phys. 96, 1, (2004)

3. T. Ohnoa, H. Yamaguchib, S. Kurodac, K. Kojimac, T. Suzukia, K. Araic, Journal of Crystal Growth, 271 (2004)

4. M. Dudley, S. Wang, W. Huang, C. H. Carter Jr, V. F. Tsvetkovz, C. Fazi, J. Phys. D: Appl. Phy. 28, (1995)

5. M. Skowronskia, S. Hab, J. Appl. Phys. 99, (2006)

6. M. Dudley, X. Huang and W. M Vetter, J. Phys. D: Appl. Phys. 36, (2003)

7. M. Katsuno, N. Ohtani, J. Takahashi, H. Yashi, M. Kanaya, Jpn. J. Appl. Phys. 38, (1999)

8. J. Chaudhuri, R. Thokala, J.H. Edgar, B.S. Sywe, Thin Solid Films 274, (1996)

9. G. R. Fisher, P. Barnes, J. F. Kelly, J. Appl. Cryst. 26, (1993).

10. D.R. Black, G. G. Long, X-Ray Topography. (NIST Special Publication 960-10 2004) 